\title{
TRANSMISSION PROBLEMS IN FUNCTION THEORY
}

\author{
BY WALTER KOPPELMAN ${ }^{1}$
}

Communicated by I. J. Schoenberg, July 3, 1961

In the year 1908, J. Plemelj [8] published an ingenious solution of the very difficult Hilbert problem for systems of analytic functions. His proof, which utilizes the theory of Fredholm integral equations, may also be found in the appendix of Muskhelishvili's monograph on singular integral equations [7]. Since Plemelj's solution of this important problem is of a fairly complicated nature, we feel that, in the light of recent advances in the theory of elliptic partial differential equations $[1 ; 3 ; 9 ; 10]$ and linear analysis $[4 ; 5]$, it is worthwhile to present a new proof which, while it is perhaps technically complicated, is conceptually much simpler than that given by Plemelj. Our approach, which is based on a continuity method, will be outlined in this note. We shall in fact discuss a general class of transmission problems, which includes the Hilbert problem mentioned above. Detailed proofs will be presented in another publication.

1. Statement of the problem. We shall pattern our formulation of the problem after that proposed in a preceding paper [6]. Accordingly, we denote by $R$ a given closed Riemann surface of genus $h$, and by $L$, a system $L_{1}, L_{2}, \cdots, L_{N}$, of simple, closed, disjoint, oriented regular curves with continuously turning tangents. Suppose that $T^{+}(s)$ and $T^{-}(s)$ are given $2 n \times n$ matrix functions on $L$, whose entries are functions of class $C^{1}$, such that

$$
\operatorname{det}\left(T^{+}, \bar{T}^{-}\right) \neq 0
$$

for all points on $L$. Let $F d \bar{z}$ be a given square integrable $n \times 1$ conjugate matrix differential on $R$ (i.e., $F d \bar{z}$ is to be invariant under conformal transformations). Denoting left and right boundary values on $L$ of an $n \times 1$ matrix function $W$ on $R-L$ by $W^{+}$and $W^{-}$respectively, we state our problem as follows:

Find all strong solutions of the transmission problem

$$
\begin{aligned}
W_{\mathrm{z}} & =F, \\
\operatorname{Re} \bar{T}^{+} W^{+} & =\operatorname{Re} \bar{T}^{-} W^{-} .
\end{aligned}
$$

The concept of "strong solution" is defined, for example, in [10], and will become clear from the discussion below.

1 This research was supported by the National Science Foundation under grant No. NSF-G14445. 
2. The adjoint problem. In order to formulate an appropriate adjoint problem, we set

$$
T=\left(T^{+}, \bar{T}^{-}\right) .
$$

According to the hypothesis (1.1), $T$ is a nonsingular, $2 n \times 2 n$ matrix function. We then form $T^{-1}$ and define two $n \times 2 n$ matrix functions $S^{+}$and $S^{-}$through the relation

$$
T^{-1}=\left(\begin{array}{l}
\bar{S}^{+} \\
S^{-}
\end{array}\right)
$$

The homogeneous adjoint problem will now consist of finding $n \times 1$ matrix differentials $V d z$ (i.e., $V d z$ is to be invariant under conformal transformations), which are strong solutions of the problem

$$
V_{z}=0, \quad \operatorname{Re} S^{+\prime} V^{+} \frac{d z}{d s}=\operatorname{Re} S^{-\prime} V^{-} \frac{d z}{d s},
$$

where, as usual, the matrices $S^{ \pm \prime}$ are obtained from the matrices $S^{ \pm}$ by the transposition of columns and rows.

3. Coercive inequalities. In order to state the coercive inequalities needed, we shall introduce certain norms. In the subsequent discussion, a function will be called piecewise smooth, if it is of class $C^{1}$ on $R-L$, and possesses continuously differentiable left and right boundary values on $L$. A similar terminology will also be used for differentials and conjugate differentials.

We first suppose that $g$ is a given, piecewise smooth coefficient of a differential on $R .^{2}$ For vector functions $W$ on $R$, we then set

$$
\|W\|_{0}^{2}=\iint_{R}|W|^{2}|g|^{2} d x d y
$$

and

$$
\|W\|_{1}^{2}=\|W\|_{0}^{2}+\iint_{R}\left\{\left|W_{x}\right|^{2}+\left|W_{\nu}\right|^{2}\right\} d x d y .
$$

For differentials $V d z$ or conjugate differentials $V d \bar{z},\|V\|_{0}$ will denote the usual $L^{2}$ norm of this quantity. We shall also utilize certain boundary norms, \langle\rangle$_{1 / 2}$, which have been introduced in $[1 ; 9]$ and whose properties are derived there. We shall not explicitly define these norms here and we shall only say that the norms of boundary values of functions with finite 1-norms (as defined in (3.2)), are

2 We also assume $g \neq 0$ on $R$. 
dominated by a constant multiple of the 1-norm of the function, where the constant depends essentially only on the geometrical aspects of the given transmission problem.

We now state

THEOREM 3.1. There exists a constant $K$, independent of $W$, such that

$$
\|W\|_{1} \leqq K\left(\left\|W_{\overline{\mathbf{n}}}\right\|_{0}+\left\langle\operatorname{Re}\left(\bar{T}^{+} W^{+}-\bar{T}^{-} W^{-}\right)\right\rangle_{1 / 2}+\|W\|_{0}\right)
$$

for all piecewise smooth $n \times 1$ vector functions $W$ on $R$.

From this theorem, we immediately have

CoRollary 3.1. For all piecewise smooth vector functions $W$ satisfying (1.3),

$$
\|W\|_{1} \leqq K\left(\left\|W_{\bar{z}}\right\|_{0}+\|W\|_{0}\right)
$$

4. The general existence theorems. In the usual way, we now form a real Hilbert space $H^{0}$ using the functions $W$, with $\|W\|_{0}<\infty$, together with the scalar product

$$
\left(W_{1}, W_{2}\right)_{0}=\operatorname{Re} \iint_{R} W_{1}^{\prime} \bar{W}_{2}|g|^{2} d x d y .
$$

Using the scalar product

$$
(W, U)_{1}=(W, U)_{0}+\operatorname{Re} \iint_{R}\left\{W_{x}^{\prime} \bar{U}_{x}+W_{y}^{\prime} \bar{U}_{y}\right\} d x d y
$$

we may also form the real Hilbert space $H^{1}$, which is to be the completion, with respect to the 1-norm, of the linear manifold of piecewise smooth functions $W$ on $R$. Concerning the spaces $H^{0}$ and $H^{1}$, we may state

Lemma 4.1 (F. Rellich). The natural imbedding of $H^{1}$ into $H^{0}$ is a compact linear transformation.

Finally, we denote by ${ }_{1} H^{0}$ the real Hilbert space of square integrable conjugate vector differentials on $R$.

We now distinguish a subspace $D$ of $H^{1}$, which is to consist of the completion with respect to the 1-norm, of the linear manifold of piecewise smooth functions $W$, satisfying (1.3). Viewed in $H^{0}$, the elements of $D$ form a dense linear manifold in $H^{0}$. We may now extend the operation $\partial / \partial \bar{z}=(\partial / \partial x+i \partial / \partial y) / 2$ by means of its continuity with respect to the 1-norm, as a mapping of elements of $H^{1}$ into ${ }_{1} H^{0}$, to all functions of $D$. We shall denote by $A$ the resulting operator with domain $D$. Of course, the elements of the domain $D$ are then pre- 
cisely all possible strong solutions of the problem (1.2)-(1.3). A similar discussion applies to the adjoint problem. Concerning this operator $A$, we state

THEOREM 4.1. As a linear transformation of $H^{0}$ into ${ }_{1} H^{0}$ with domain $D$, the operator $A$ is closed, has a finite-dimensional null space, and possesses a closed range of finite codimension in ${ }_{1} H^{0}$. Further, a coniugate differential $F d \bar{z}$ lies in the range of the operator $A$ if, and only if

$$
\operatorname{Re} \iint_{R} V^{\prime} F d x d y=0
$$

for every strong solution $V$ of problem (2.3).

The proof of this theorem is similar to the proofs dealing with existence theorems for normal boundary value problems for elliptic differential equations $[3 ; 10]$.

5. Homotopy classification of transmission problems. Given two transmission problems

$$
W_{\bar{z}}=F, \quad \operatorname{Re} \bar{T}_{\nu}^{+} W^{+}=\operatorname{Re} \bar{T}_{\nu}^{-} W^{-}, \quad \nu=0,1,
$$

together with all hypotheses of $\$ 1$, we shall say that these two problems are homotopically equivalent, if there exists a one-parameter family of transmission problems

$$
W_{\bar{z}}=F, \quad \operatorname{Re} \bar{T}_{t}^{+} W^{+}=\operatorname{Re} \bar{T}_{\bar{t}}^{-} W^{-}, \quad 0 \leqq t \leqq 1,
$$

each of which satisfies the conditions of $\S 1$, with $T_{t}^{ \pm}(s),(d / d s) T_{t}^{ \pm}(s)$ continuous in $(s, t)$, and $\left.T_{t}^{ \pm}\right|_{t=0}=T_{0}^{ \pm},\left.T_{t}^{ \pm}\right|_{t=1}=T_{1}^{ \pm}$. Homotopy classes of matrices have recently been investigated by Boyarskir [2], and his results may, after only minor modifications, be adapted to our problem. In order to state the results in a concise form, we make two definitions. By the characteristic $\kappa$ of problem (1.2)-(1.3), we shall mean the integer

$$
\frac{1}{2 \pi}\left[\arg \operatorname{det}\left(T^{+}, \bar{T}^{-}\right)\right]_{L}
$$

By the $j$-characteristic $\kappa_{j}$, we shall mean the integer

$$
\frac{1}{2 \pi}\left[\arg \operatorname{det}\left(T^{+}, \bar{T}^{-}\right)\right]_{L_{j, j-1,2, \cdots, N}}
$$

We now have 
THEOREM 5.1. The two problems (5.1) are homotopically equivalent if, and only if all of their $j$-characteristics coincide.

6. The index of transmission problems. If $\alpha$ denotes the number of linearly independent strong solutions of the homogeneous problem (1.2)-(1.3) $\left(W_{z}=0\right)$, and $\beta$ denotes the number of linearly independent strong solutions of problem (2.3), then, by the index $k$ of problem (1.2)-(1.3) we shall mean the integer

$$
k=\alpha-\beta .
$$

We now consider a family $T_{t}^{ \pm}, 0 \leqq t \leqq 1$ of regular boundary conditions, as in $\S 5$. With the aid of the results of $\S \S 3,4$, it is now possible to define a linear operator $M_{t}$ of $H^{1}$ into itself, which is bounded in the 1-norm, such that $1-M_{t}$ maps the domain $D_{t}$ of the operator $A_{t}$ corresponding to the boundary conditions $T_{t}^{ \pm}$into $D_{0}$, the domain of $A_{0}$. Further, Theorem 3.1 may be used to show that the norm of $M_{t}$ satisfies the relation

$$
\lim _{t \rightarrow 0}\left\|M_{t}\right\|_{1}=0 \text {. }
$$

Consequently, for sufficiently small $t, 1-M_{t}$ has an inverse, which we write in the form $1+\hat{M}_{t}$. It is clear that

$$
\lim _{t \rightarrow 0}\left\|M_{t}\right\|_{1}=0 \text {. }
$$

For $W$ in $D_{0}$, we may now obtain the inequality

$$
\left\|\frac{\partial}{\partial \bar{z}} \hat{M}_{t} W\right\|_{0} \leqq\left\|\hat{M}_{t}\right\|_{1} K_{0}\left(\left\|A_{0} W\right\|_{0}+\|W\|_{0}\right) .
$$

If we set $B_{t}=(\partial / \partial \bar{z}) \hat{M}_{t}$, we see from (6.3) that the operator $B_{t}$ is $A$ bounded, in the terminology of [4], and that its $A$-norm is small, provided that $t$ is small. We may now apply theorems in the invariance of the index $[4 ; 5]$ to the operators $A+B_{t}$. Together with Theorem 5.1 and the fact that $1+\hat{M}_{t}$ is a continuously invertible mapping of $D_{0}$ onto $D_{t}$, we may now derive

Theorem 6.1. The index $k$ of problem (1.2)-(1.3) is given by the relation

$$
k=2(\kappa-n(h-1)) .
$$

Formula (6.4) is obtained by connecting the given problem with a simple problem, whose index may be established through the Riemann-Roch theorem. 


\section{BIBLIOGRAPHY}

1. S. Agmon, The coerciveness problem for integro-differential forms, J. Analyse Math. vol. 6 (1958) pp. 183-223.

2. B. V. Boyarski1, Homotopy classes of matrix functions, Soobšc. Akad. Nauk Gružin. SSR vol. 21 (1958) pp. 263-269.

3. F. E. Browder, Estimates and existence theorems for elliptic boundary value problems, Proc. Nat. Acad. Sci. U.S.A. vol. 45 (1959) pp. 365-372.

4. I. C. Gohberg and M. G. KreIn, The basic propositions on defect numbers, root numbers and indices of linear operators, Uspehi Mat. Nauk (N. S.) vol. 12 (1957) pp. 43-118; Amer. Math. Soc. Translations Series 2, vol. 13, 1960, pp. 185-264.

5. T. Kato, Perturbation theory of nullity, deficiency and other quantities of linear operators, J. Analyse Math. vol. 6 (1958) pp. 261-322.

6. W. Koppelman, Singular integral equations, boundary value problems and the Riemann-Roch theorem, J. Math. Mech. vol. 10 (1961) pp. 247-277.

7. N. I. Muskhelishvili, Singular integral equations, P. Noordhoff, Groningen, 1953. (Translated from the Russian).

8. J. Plemelj, Riemannsche Funktionenscharen mit gegebener Monodromiegruppe, Monatsh. Math. Phys. vol. 19 (1908) pp. 211-245.

9. M. Schechter, General boundary value problems for elliptic partial differential equations, Comm. Pure Appl. Math. vol. 12 (1959) pp. 457-486.

10. - Remarks on elliptic boundary value problems, Comm. Pure Appl. Math. vol. 12 (1959) pp. 561-578.

The University of Pennsylvania 